\title{
Harvesting Implicit Group Attitudes and Beliefs From a Demonstration Web Site
}

\author{
Brian A. Nosek \\ Yale University
}

\author{
Mahzarin R. Banaji \\ Harvard University
}

\author{
Anthony G. Greenwald \\ University of Washington
}

\begin{abstract}
Respondents at an Internet site completed over 600,000 tasks between October 1998 and April 2000 measuring attitudes toward and stereotypes of social groups. Their responses demonstrated, on average, implicit preference for White over Black and young over old and stereotypic associations linking male terms with science and career and female terms with liberal arts and family. The main purpose was to provide a demonstration site at which respondents could experience their implicit attitudes and stereotypes toward social groups. Nevertheless, the data collected are rich in information regarding the operation of attitudes and stereotypes, most notably the strength of implicit attitudes, the association and dissociation between implicit and explicit attitudes, and the effects of group membership on attitudes and stereotypes.
\end{abstract}

Among the most fundamental groups to which humans belong are their gender, race/ ethnicity, age, socioeconomic status, religion, nationality, and political and intellectual orientations. Such groups typically contain large numbers of others, often spread across the world, and direct interpersonal contact with only a small subset of these individuals. Yet such membership, the mere act of belonging, can determine psychological, social, and economic fates in significant ways. Whether one is African or European, female or male, rich or poor, Catholic or Protestant, Pakistani or Indian determines not only the knowledge and feelings

Brian A. Nosek, Department of Psychology, Yale University; Mahzarin R. Banaji, Department of Psychology, Harvard University; Anthony G. Greenwald, Department of Psychology, University of Washington.

This research was supported by National Science Foundation Grants SBR-9422241, SBR-9422242, SBR-9709924, and SBR-9710172 and National Institute of Mental Health Grants MH-41328, MH-01533, and MH-57672. Special thanks go to Richard Hackman for his comments on a draft of this article and to Joseph Bella, Josh Book, Henry Kaufman, David McNellis, and Wil Cunningham for their contributions to the Web site.

Correspondence concerning this article should be addressed to Brian A. Nosek, Department of Psychology, Yale University, P.O. Box 208205, New Haven, Connecticut 06520-8205. E-mail: brian.nosek@yale.edu that constitute the individual but also the manner in which that person, as a representative of the group, is perceived and treated in the larger sociopolitical sphere. The contribution of psychology has been to study exactly these processes: What lies within individual minds in the form of attitudes, knowledge, beliefs, and identity? How do the processes underlying group cognition influence perception and behavior? In this article, we provide evidence about group cognition, that is to say, attitudes and knowledge about social groups. In the context of other articles in this issue, our work is unique in two ways. First, we examined the contents of group cognition that, at least to some extent, are not within an individual's conscious control. Second, we obtained evidence of group cognition from drop-in respondents at a Web site that in 3.5 years has produced over 1.5 million completed tasks, each measuring some aspect of self or group cognition (e.g., age, race, gender attitudes, and knowledge). Here we report on a subset of those data, keeping in mind both the limitations of such a method for gathering data and the extraordinary opportunities it presents for assessing the mental processes that underlie social behavior. To our knowledge, this is the first large-scale Web-based data collection on attitudes toward and knowledge about social groups. 
In September 1998 we designed and launched a Web site at which drop-in visitors could take simplified versions of Implicit Association Tests (IATs; Greenwald, McGhee, \& Schwartz, 1998) that had been in laboratory use for several years. The Web site was set up on the model of an interactive exhibit at a science museum. In a few minutes, visitors had the opportunity to complete a measure of implicit attitude or belief (stereotype), after optionally responding to some demographic items and a single-item self-report index of the attitude or belief. It was expected that most respondents would have the opportunity to experience what we and many laboratory subjects have - the uncontrollability of automatic attitudes and stereotypes, even those that are consciously disavowed. As the Web site accumulated data, respondents also had the opportunity to view aggregate patterns in the form of graphs or tables containing summary statistics of the performance of large numbers of visitors to the Web site and to learn about research on implicit attitudes and beliefs toward social groups more generally. A total of 600,000 tests were completed between October 1998 and April 2000, a response that far outstripped expectation.

A goal of this work was to provide the necessary experience for future Web-based research that will more adequately meet the criteria for full-scale psychological experiments. The vast possibilities contained in Web-based research for advancing psychological science, while educating the funding public, motivated the creation of this demonstration Web site.

Although the main purpose of the Web site remains educational, the experience of creating and maintaining the site has provided an opportunity to learn about implicit attitudes and stereotypes toward social groups from a large sample. We recognize that drop-in respondents to the Web site are by no means a random sample; nevertheless, their diversity was expected to, and did, exceed that of the college samples on which laboratory data using these tasks had been collected. In particular, the present demonstration site can potentially provide insights into the veracity of Web data, and of response time data in particular, by allowing study of their consistency with laboratory data and with theory.

\section{Implicit Social Cognition and the IAT}

For the past two decades, psychologists have used measures of prejudice and stereotypes that operate outside conscious awareness or conscious control (e.g., Banaji \& Greenwald, 1995; Banaji \& Hardin, 1996; Bargh, Chen, \& Burrows, 1996; Chartrand \& Bargh, 1999; Devine, 1989; Dovidio, Kawakami, Johnson, Johnson, \& Howard, 1997; Fazio, Jackson, Dunton, \& Williams, 1995; Gilbert \& Hixon, 1991; Greenwald et al., 1998; Perdue \& Gurtman, 1990). The IAT Web site demonstrates just one of many measures, the Greenwald et al. (1998) Implicit Association Test, which is easy to administer and produces large effects. In addition, this test provides a palpable experience of the greater ease of some associations (e.g., old with bad) over others (e.g., young with bad) and the relative uncontrollability of such associations. As a result, it provides a more direct experience of potential dissociations between one's conscious and unconscious attitudes and beliefs (much like the experience evoked by other response competition tasks, such as the Stroop test).

This report describes results of 600,000 implicit attitude and stereotype tests obtained in the first 19 months of operation of the IAT demonstration site, including their relationship to single-item explicit attitude and stereotype measures. The results also shed light on methodological questions regarding the IAT and Web-administered research.

\section{Method}

\section{Respondents}

Recruitment. Respondents were neither preselected nor targeted for participation. Recruitment occurred through four known channels: media coverage, links from other sites, search engines, and word of mouth, with media coverage having the most significant effect on response rate. For example, over 150,000 visits to the IAT site were recorded in the 5 days following televised coverage on the NBC program Dateline (March 19, 2000) and a Discovery Channel show on prejudice (March 20, 2000).

Demographics. Demographic data were assessed by optional self-report questions that 
were presented immediately prior to the IATs. Of the approximately $90 \%$ of individuals who chose to report such information, $62 \%$ $(358,008)$ were female and $38 \%(218,450)$ were male; $37 \%$ were under 23 years of age, $55 \%$ were ages $23-50$, and $8 \%$ were over $50 ; 1 \%$ were American Indian, 5\% Asian, 9\% Black, 4\% Hispanic, $77 \%$ White, and 4\% "other."

\section{Materials and Apparatus}

The Web IAT was constructed using Java Applet and common gateway interface (CGI) technology. Once downloaded, the program used the respondent's computer to present stimuli and measure responses latencies. ${ }^{1}$ The server analyzed the respondent's data and reported a result of the test to the respondent's computer.

Implicit tasks. Nine IAT measures and accompanying brief questionnaires were available at the Web site at different times starting in late September 1998: (a) race attitude, one using African American and European American first names and a second using morphed faces, and the attribute of good-bad; (b) age attitude, one using first names and a second using morphed faces, and the attribute of good-bad; (c) gender-career stereotype, measuring the association of male-female terms with career and family terms; (d) gender-science stereotype, measuring the association of male-female terms with science and liberal arts terms; (e) selfesteem, measuring attitudes toward self versus other; (f) math-arts attitude; and (g) Election 2000 preference, measuring attitudes toward candidates in the U.S. presidential primaries of 2000. See Appendix A for a complete list of the stimuli and category labels used in all tasks. Because the focus of this article is on groups, the math-arts attitude, self-esteem, and Election 2000 IATs are not discussed in detail. For completeness, summary data for those tasks are included in the tables. ${ }^{2}$

Explicit measure and demographic information. Before performing each IAT, respondents completed a single-item (5-point scale) measure of explicit attitude or belief toward the attitude or belief object and provided minimal demographic information. In addition, they were requested to provide answers to task-appropriate questions, such as "Please rate your attitude toward science" for the gender-science task (see Appendix B for a full list of all ques- tions associated with each IAT). Finally, respondents indicated their age, gender, ethnicity, education level, and state or country of residence, the population of their hometown, and previous experience with the IAT (i.e., number of previous such tests taken). ${ }^{3}$

\section{Procedure}

Respondents first reviewed preliminary information about the IAT and then pressed a link to continue if they wished to do so. Second, respondents chose a task from a list of four to six that were available. Third, respondents reported their explicit attitude in response to an item that was worded to capture the comparison of concepts (e.g., preference for young vs. old) that was used in the upcoming IAT measure and then reported their demographic characteristics. Fourth, respondents read instructions for the IAT and proceeded to complete it. Finally, respondents were provided with an IAT result, were shown how their result compared with those of others who had completed the same task, and were given the opportunity to review additional information available at the site on implicit social cognition. Each such sequence, including questionnaire and IAT, took 5-10 min

\footnotetext{
${ }^{1}$ For security purposes, applets do not have access to many of the functional components of a client's computer. As such, millisecond timing is based on the machine's internal clock that provides more coarse response times than are typical in this type of research. Apple Macintoshes have an error window of $16.7 \mathrm{~ms}$ per response, and PC machines have a window of between 50 and $60 \mathrm{~ms}$. Though imperfect, these windows have little effect on the observed results because IAT effects are large and many (40) trials are used per block.

${ }^{2}$ The IAT is a relatively new measure, and questions concerning its methodological properties are of interest. The demonstration Web site provides the advantage of very large numbers of observations that can be applied to evaluate the methodological properties of the IAT. With the large samples available, the absence of effects can be interpreted more confidently than in a typical laboratory study, in which low statistical power obliges caution in interpreting null results. With the Web data, we have examined (a) the relationship between error rates and the IAT effect, (b) the relationship between speed to respond and the IAT effect, (c) the relationship between experience with the IAT and the IAT effect, (d) the effect of order of presenting tasks within the IAT, and (e) the effect of respondent handedness on IAT effects. A summary of these analyses is available at http://www.briannosek.com.

${ }^{3}$ To sample some of the procedures described here more directly, visit www.yale.edu/implicit.
} 
to complete. The preliminary information included several warnings cautioning respondents about the potential discomfort that may be produced by the experience and described the limitations of the procedure itself; participants were reminded that they were under no obligation to complete the task after starting it.

\section{Caveats About Web-Based Data}

Data collected via this Web site involved the use of simple tasks with no attempt to undertake the testing of new hypotheses. These data should therefore be considered as open-survey results with two important constraints: (a) Respondents were self-selected and (b) respondents could perform multiple tasks and/or the same task multiple times.

Self-selection. This challenge for psychological research (including laboratory research that provides even minimal information regarding the nature of the experiment) is especially apparent for Web-based research. Web respondents likely chose to participate for a variety of reasons: an interest in psychological research, a positive (or negative) orientation toward the topics or methods represented at this site, a recommendation from a friend, or a recommendation or requirement in a school or college course. These characteristics assuredly do not provide random sampling, but they do result in a diversity of respondents. However, the chief value of these data may be the large number of respondents available for each task.

Multiple submissions from a single respondent. Because participation at the IAT Web site was open and uncontrolled, individual respondents could choose to participate as many times as they wished. Multiple data points from single respondents obviously pose serious problems for data interpretation. Although the large number of respondents makes it unlikely that the effects of multiple submissions would have major impact on observed results, we created a conservative data set in which only participants who reported having performed no more than one task were included. Patterns of data were extremely consistent between this conservative data set and the full data set. ${ }^{4}$ Hence, the analyses reported here used the full data set.

\section{Analysis}

Data reduction. Analysis of IAT data followed the procedure outlined by Greenwald et al. (1998). Respondents who did not follow instructions or did not perform the task up to minimum performance criteria were removed from the analysis. These criteria eliminated only the most extreme responses on the IAT-those that were too slow or those that revealed substantial errors in classification. Respondents whose average latencies for either critical block were over $1,800 \mathrm{~ms}$ or whose average overall latency was above $1,500 \mathrm{~ms}$ were also removed from the analysis ( $2 \%$, or 10,723 respondents). Respondents who made in excess of $25 \%$ errors in any single critical block were removed from the analysis (13\%, or 84,717 respondents). In total, $85 \%(541,696)$ of the total sample provided interpretable data. ${ }^{5}$ We calculated the IAT effect by calculating the difference score between average response latencies for the two critical blocks of each task (e.g., Black with good - Black with bad; see Greenwald, Nosek, \& Banaji, 2002, for an improved scoring algorithm for the IAT).

Data reporting. With the large samples that are available, ordinary tests of significance have little meaning (e.g., in many instances, Pearson's $r$ correlations of .01 or less were statistically significant). As such, we report not significance tests but rather mean effects and effect sizes (in the form of Pearson's $r$ correlations or Cohen's $d$; Cohen, 1988).

\footnotetext{
${ }^{4}$ The full data set was also compared with a second, more conservative data set in which a data point was included only if the participant reported performing only one or fewer tasks and if only one submission per Internet protocol (IP) address was included. Again, results were very consistent across data sets. The only circumstance in which a notable change occurred is summarized in footnotes 7 and 10 .

${ }^{5}$ The fact that $15 \%$ of the sample did not provide interpretable data should not be taken as a reflection on the abilities of the respondents to perform the task. Rather, it points out the difficulty of translating research performed in the traditional laboratory to that conducted using the Internet. For Internet research, the physical absence of the experimenter increases the difficulty of ensuring that procedural instructions are clear to all respondents.
} 
Table 1

Implicit and Explicit Attitudes for Each of the Nine Tasks Available at the IAT Web Site Between October 1998 and April 2000

\begin{tabular}{|c|c|c|c|c|c|c|c|c|}
\hline \multirow[b]{2}{*}{ Task } & \multirow[b]{2}{*}{ Total $N$} & \multirow[b]{2}{*}{ Interpretable $n$} & \multicolumn{2}{|c|}{ IAT effect } & \multirow[b]{2}{*}{ Cohen's $d$} & \multicolumn{2}{|c|}{ Explicit } & \multirow[b]{2}{*}{ Cohen's $d$} \\
\hline & & & $M$ & $S D$ & & $M$ & $S D$ & \\
\hline Race attitude (faces) & 192,364 & 160,857 & 158 & 224 & 0.71 & 0.30 & 0.83 & 0.36 \\
\hline Race attitude (names) & 70,807 & 60,197 & 187 & 213 & 0.88 & 0.24 & 0.90 & 0.27 \\
\hline Age attitude (faces) & 79,888 & 68,144 & 196 & 198 & 0.99 & 0.41 & 0.81 & 0.51 \\
\hline Age attitude (names) & 53,125 & 42,641 & 301 & 212 & 1.42 & 0.29 & 1.04 & 0.28 \\
\hline Gender-science & 73,117 & 61,228 & 136 & 190 & 0.72 & 0.49 & 0.67 & 0.73 \\
\hline Gender-career & 42,105 & 38,797 & 95 & 133 & 0.72 & 0.28 & 0.56 & 0.50 \\
\hline Self-esteem & 50,514 & 44,907 & 173 & 156 & 1.12 & 0.47 & 1.20 & 0.39 \\
\hline Math-arts attitude & 31,991 & 28,108 & 153 & 186 & 0.82 & 0.47 & 1.43 & 0.33 \\
\hline Election 2000 attitude & 43,225 & 36,840 & 29 & 215 & 0.14 & 0.26 & 1.55 & 0.17 \\
\hline
\end{tabular}

Note. Main effects are in milliseconds. Positive values indicate preference for White over Black (race tasks), preference for young over old (age tasks), association of male with science and female with liberal arts (gender-science), association of male with career and female with family (gender-career), preference for self over other (self-esteem), preference for math over arts (math-arts attitude), and preference for Gore over Bush (Election 2000). For the Election 2000 task, only those data for which Gore and Bush were compared are presented $(n=22,980)$. IAT $=$ implicit association test.

\section{Results and Discussion}

Table 1 presents a summary of the IAT effects for the nine tasks presented at the Web site. The following sections refer to specific IATs in this table and summarize results of particular relevance to the measure being discussed.

\section{Race Attitudes}

In the race IATs, respondents classified Black and White faces or names while classifying words of positive or negative valence. Overall, the observed IAT effect revealed respondents' automatic preference for White relative to Black. For both name $(d=0.71)$ and face $(d=0.88)$ tasks, participants showed an automatic preference for White over Black. Although participants likewise showed preference for White over Black on the explicit measure, the magnitude of that effect was noticeably smaller $(d \mathrm{~s}=0.27$ and 0.36 , respectively). These results replicate data collected using traditional laboratory procedures (Greenwald et al., 1998; Mitchell, Nosek, \& Banaji, 2001).

An immediate question arises regarding the performance of Black compared with White respondents as well as those from other ethnic groups. Traditional laboratory data have been limited in the availability of Black American subjects, and hence Web data are of particular interest. Figure 1 presents implicit and explicit race attitudes in the form of effect sizes (Cohen's $d$ ) separately for Black and White respondents. ${ }^{6}$ On the explicit measure, White respondents showed a preference for White over Black $(d=0.59)$, but Black respondents showed an opposite and even stronger preference for Black over White $(d=0.80)$. This strong explicit liking reported by Black respondents for their own group stands in sharp contrast to performance on the implicit measure. Unlike White respondents, who showed a strong preference for White over Black $(d=0.83)$, Black respondents showed a weak preference for White over Black $(d=0.16) .{ }^{7}$

This pattern mimics the laboratory data obtained with Black and White college students (Banaji, Greenwald, \& Rosier, 1997), in which Black students showed strong explicit liking and identification with their own social group (compared with White students), whereas a reversed pattern was observed on implicit mea-

\footnotetext{
${ }^{6}$ Effects are similar for the race name task. See Table 2.

${ }^{7}$ Scores for Black respondents on the race task were notably different between the full data set and the conservative data set (unique internet protocol [IP] addresses). For both the face task and the name task, the conservative data set showed Black respondents to have less preference for White over Black than is apparent in the full data set: fullset: face $=39 \mathrm{~ms}(d=0.16)$, name $=63 \mathrm{~ms}(d=0.28)$; conservative set: face $=-26 \mathrm{~ms}(d=0.12)$, name $=29 \mathrm{~ms}$ $(d=0.14)$. In fact, Black respondents in the face task switch from showing a slight preference for White over Black to a slight preference for Black over White.
} 


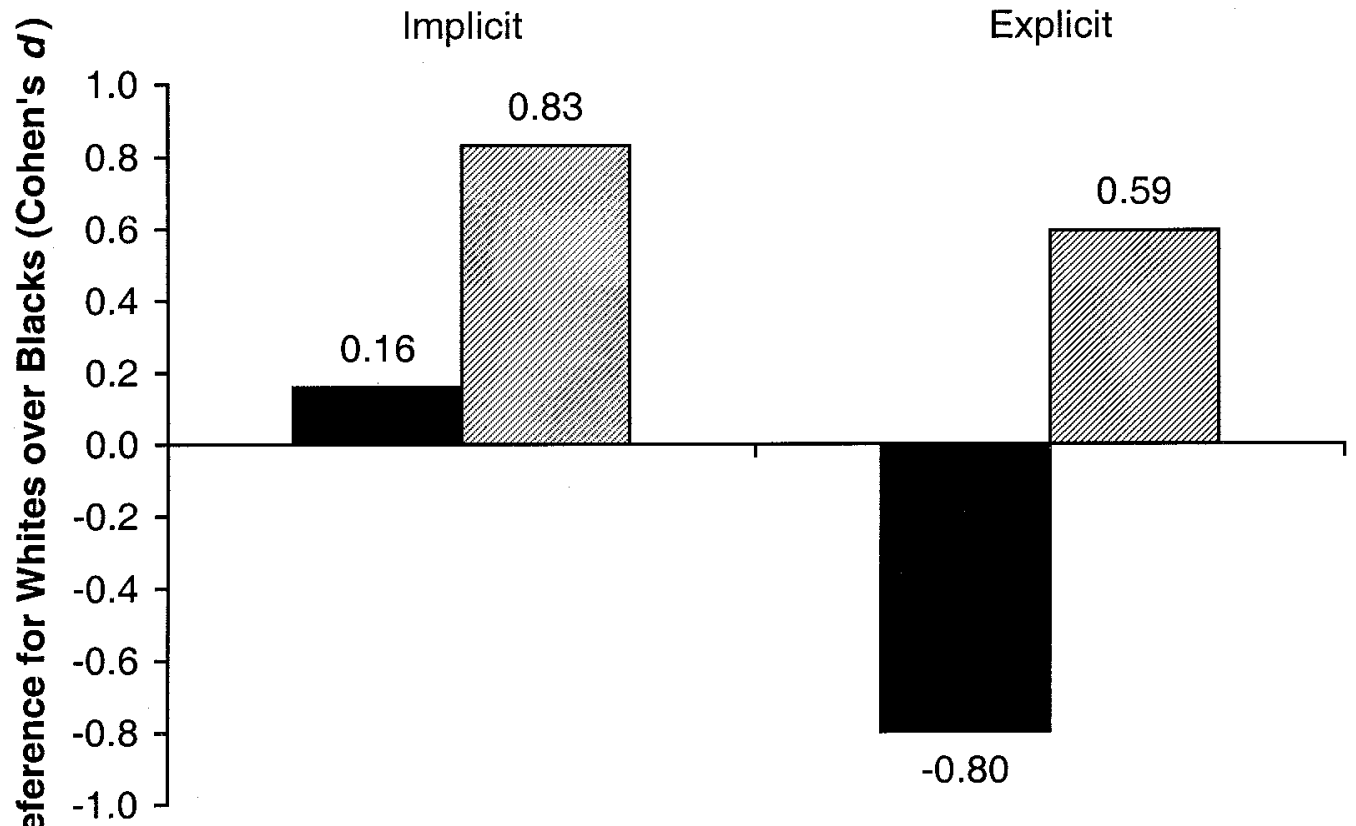

\section{Blacks Whites}

Figure 1. Implicit and explicit attitudes toward Whites versus Blacks by respondent race (race face task; White respondents, $n=103,316$, Black respondents, $n=17,510$ ). Positive Cohen's $d$ s reflect a preference for White over Black; negative values reflect a preference for Black over White.

sures (with White students showing stronger implicit in-group preference). Such data raise many questions of both theoretical and political import, most obviously, the role of culturally imposed evaluations of social groups and the permeability of such evaluations into the minds of individual members of the culture, even among those who hold membership in the group (Jost \& Banaji, 1994). The meaning and consequences of this dissociation between the explicit-implicit nature of the attitude and social group (Black-White) will no doubt be a continuing focus in our research because of its potential to understand the deep reach of culturally acquired attitudes that are reflected in behavioral and brain activity (see Phelps et al., 2001).

Consistent with well-established links between political ideology and explicit attitudes (i.e., the greater one's liberal orientation, the greater the self-reported pro-Black attitude), explicit racial bias was weaker among those who reported a more liberal than conservative ideology (see Figure 2; Katz \& Hass, 1988; Katz, Wackenhut, \& Hass, 1986). A similar but weaker effect was observed on the implicit measure of racial bias. Liberal respondents demonstrated somewhat weaker implicit racial bias than did conservative respondents. These effects are consistent with recent findings suggesting that personal standards and practicing nonprejudiced responses can influence the magnitude of implicit biases (Moskowitz, Gollwitzer, Wasel, \& Schaal, 1999). For example, Cunningham, Nezlek, and Banaji (2000) have shown a relationship between rigidity in thinking, rightwing ideology, and conscious and unconscious attitudes toward a variety of social groups (i.e., Black, Jewish, poor, gay, and foreign).

\section{Age Attitudes}

In the age IAT task, respondents classified old and young names or faces paired with words 


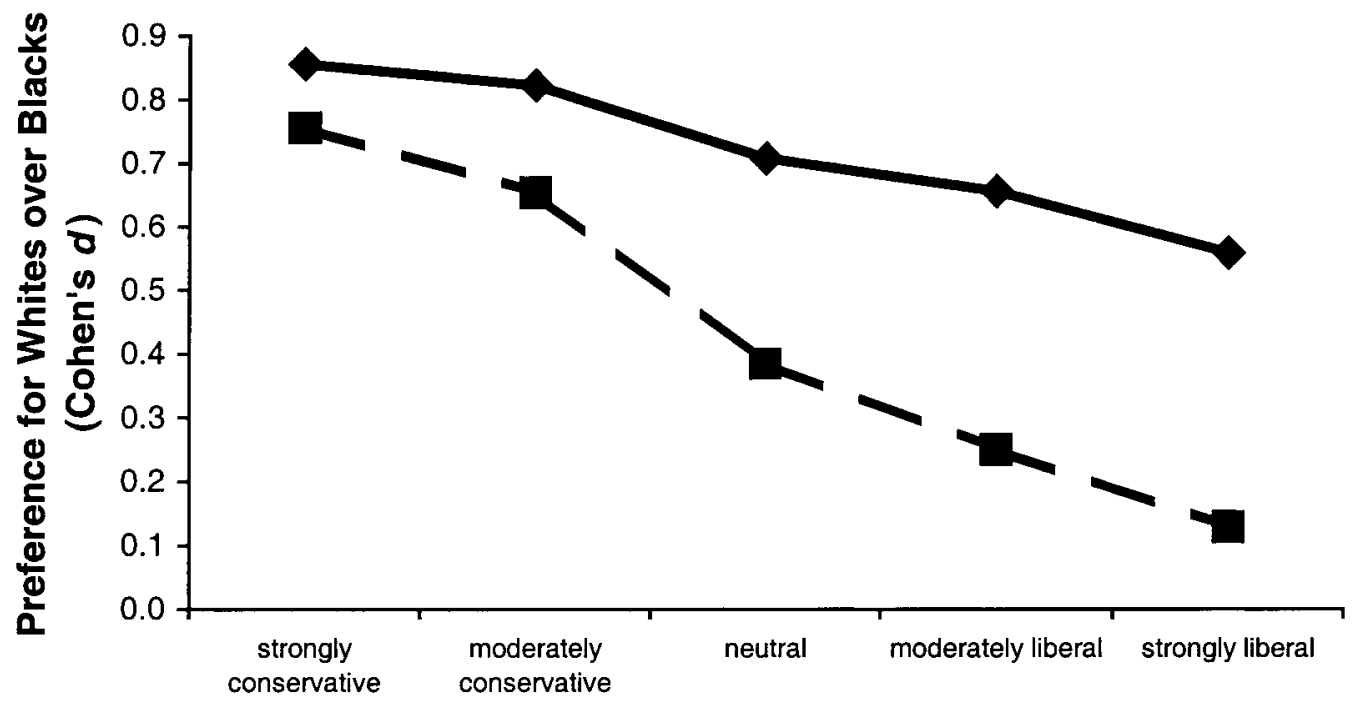

Conservatism - Liberalism

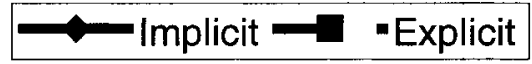

Figure 2. Implicit and explicit attitudes toward young versus old by respondent age (age face task; $n=68,144)$. Positive Cohen's $d$ s reflect a preference for young over old; negative values reflect a preference for old over young.

of positive or negative valence. The observed IAT effect indexed respondents' automatic preference for young relative to old. For both the name and face tasks, respondents showed a strong automatic preference for young over old $(d=1.42$ and 0.99 , respectively; see Table 1$)$. On explicit measures, respondents also showed liking for young over old, but the magnitude of such effects was much weaker $(d \mathrm{~s}=0.28$ and 0.51 , respectively).

Negative implicit attitudes toward old relative to young were, on average, the strongest of any obtained at the demonstration Web site. Again, the question of group membership emerges: Does being old mitigate the magnitude of the anti-old attitude? In Figure 3, implicit and explicit attitudes toward young versus old are reported as a function of respondent age. ${ }^{8}$ As expected on the basis of positive ingroup attitudes, a clear linear trend showed that increases in positive explicit attitudes toward old relative to young were commensurate with respondent age (although old was never preferred to young except for a slight old prefer- ence among respondents whose reported age was $71+$ ).

Of most interest is the lack of a corresponding increase, with respondent age, in positivity toward old on implicit attitudes. Strong negative implicit attitudes toward old were present from the youngest (8-14 years) to the oldest $(71+$ years) respondents in this Web sample. Like Black respondents on the race IAT, the elderly also self-reported greater positivity toward their own group (compared with those who are younger) without a corresponding implicit positivity for their own group.

\section{Gender Stereotypes About Science and Liberal Arts}

In the gender-science IAT, respondents classified science and liberal arts terms (e.g., physics and literature) while classifying male and female terms (e.g., he and she). The observed

\footnotetext{
${ }^{8}$ Effects are similar for the age name task.
} 

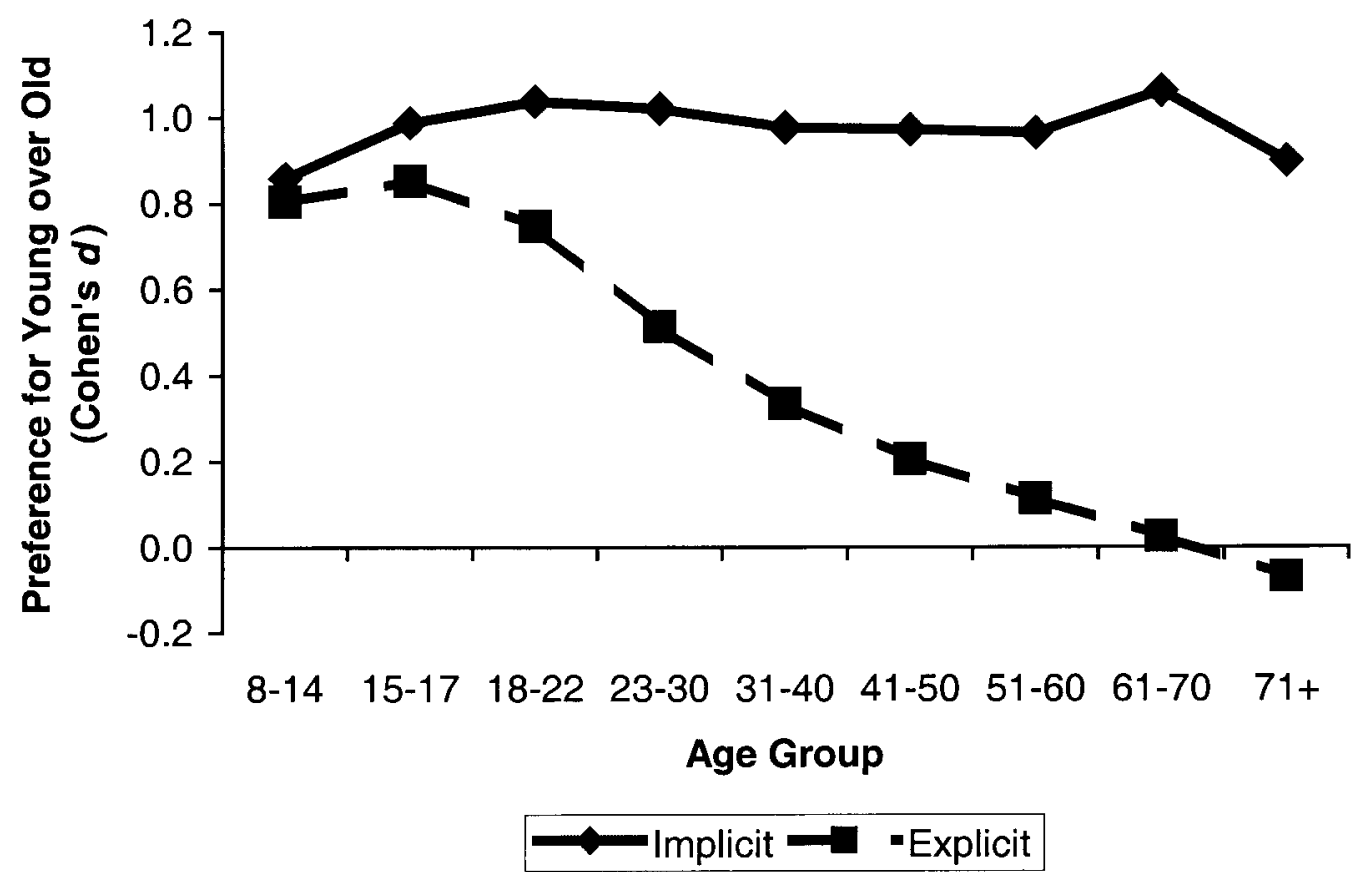

Figure 3. Implicit and explicit attitudes toward Whites versus Blacks by respondent conservatism-liberalism (race face task; $n=160,857$ ). Positive Cohen's $d$ s reflect a preference for White over Black; negative values reflect a preference for Black over White.

IAT effect indexed respondents' automatic association of male with science and female with liberal arts compared with the complementary pairings. Respondents showed robust associations of male with science and female with liberal arts $(d=0.72$; see Table 1$)$, replicating similar demonstrations in laboratory research (Nosek, Banaji, \& Greenwald, in press). Similar links of male with science and female with liberal arts were observed with explicit measures $(d=0.73)$.

Although far from equal with men, the proportional representation of women in science has been steadily increasing since the 1950s (National Science Foundation, 1996). Is this shift in representation associated with a corresponding decline in the magnitude of the stereotype observed across age cohorts? Because the demonstration site does not provide longitudinal data at this time, the best indicator of such an effect may be the magnitude of genderscience associations across age clusters. Figure 4 presents implicit and explicit stereotypes about gender and science by respondent age. Notably, both implicit and explicit stereotypes tend to be stronger among older respondents compared with younger respondents. Second, until age 50, implicit and explicit gender stereotypes about science are equivalent in magnitude. After age 50, implicit stereotypes linking males to science and females to liberal arts continue to increase with age, whereas explicit attitudes do not.

The overall relative association of male to science and female to arts is not surprising given the strong and historically skewed gender distribution in science. What is perhaps remarkable, compared with other measures, is the equally strong nature of implicit and explicit stereotypes. This result may well reveal the lack of social disapproval associated with this discrepancy.

\section{Gender Stereotypes About Career and Family}

In the gender-career IAT, respondents classified career and family terms (e.g., executive and children) while classifying male and female terms (e.g., boy and girl). The IAT measure 

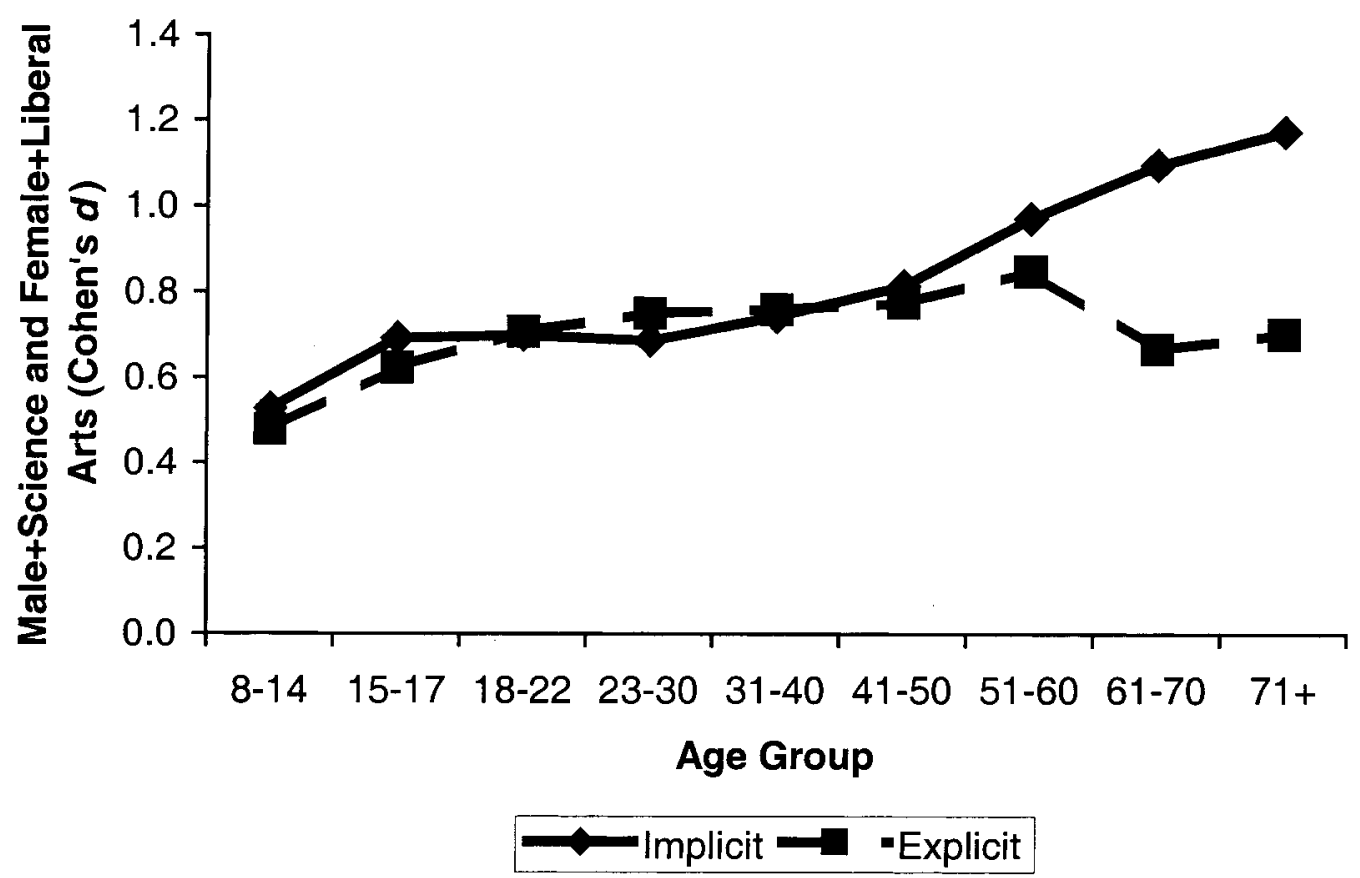

\begin{abstract}
Figure 4. Implicit and explicit stereotypes linking male with science and female with liberal arts by respondent age (men, $n=19,906$; women, $n=36,548$ ). Positive Cohen's $d$ s reflect male with science and female with liberal arts associations; negative values reflect male with liberal arts and female with science associations.
\end{abstract}

indexed respondents' automatic association of male with career and female with family compared with the complementary pairings. Respondents showed robust associations of male with career and female with family $(d=0.72$; see Table 1), replicating similar demonstrations in laboratory studies (Wang \& Banaji, 1999). Similar but slightly weaker links of male with career and female with family were observed with explicit measures $(d=0.50)$.

Both men and women linked male with career and female with family, but whereas men showed comparably strong associations on implicit and explicit measures, women showed a stronger effect than men on the implicit measure and a weaker effect than men on the explicit measure (see Figure 5). Women appear to endorse some combination of career and family for women more than men do, but they appear not to go along with that conviction at the implicit level. Rather, on the implicit measure, women show the culturally prescribed associations that link their group with family more than with career. This finding, that group member- ship does not protect against exhibiting the stereotype (Banaji \& Greenwald, 1995), suggests that such differences are more readily observable at the implicit than explicit level (a finding corroborated by race and age data in previous sections; see Banaji, 2001).

\section{Group Effects}

Gender of respondent effects. Table 2 presents IAT effects for each task separated by respondent gender. The data in Table 2 reveal that women showed slightly less negativity than did men toward socially disadvantaged race and age groups. Likewise, little to no gender differences were noted in gender-science stereotypes or gender-career stereotypes. Women showed greater negativity toward math relative to arts than men $\operatorname{did}(d=0.34)$, replicating laboratory data reported by Nosek et al. (in press).

Ethnicity comparisons. Table 2 presents IAT effects for four ethnic groups for whom substantial data were available: Asian, Black, Hispanic, and White. On both race tasks (faces 


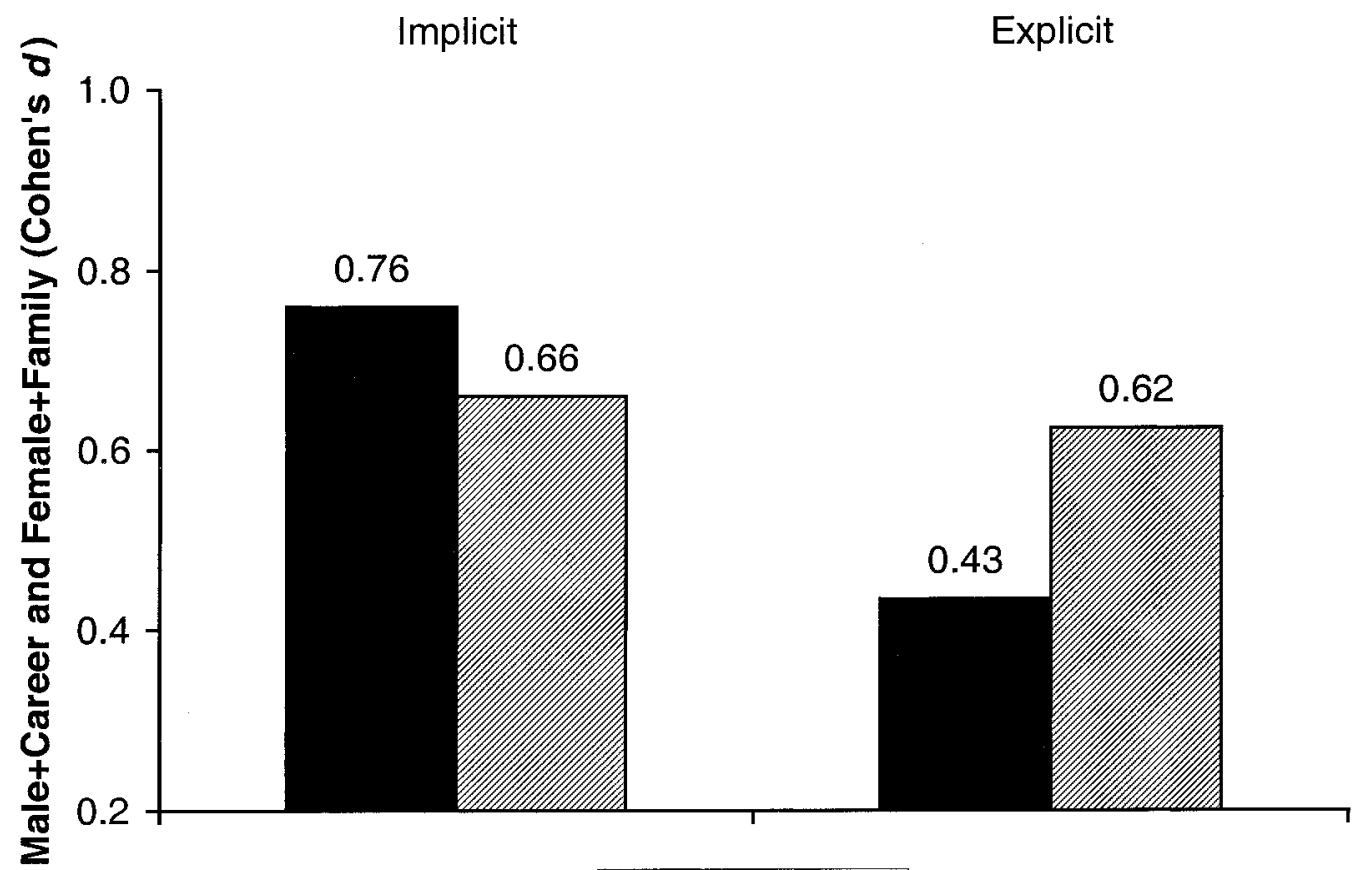

women 四en

Figure 5. Implicit and explicit stereotypes linking male with career and female with family by respondent gender ( $n=38,797)$. Positive Cohen's $d$ s reflect male with career and female with family associations; negative values reflect male with family and female with career associations.

and names), White respondents showed the strongest pro-White bias, and Black respondents showed the weakest. It is noteworthy that Asian and Hispanic respondents also showed pro-White bias at levels comparable to White respondents. On age attitudes, gender-science stereotyping, and gender-career attitudes, members of the four ethnic groups showed similar effects.

Age comparisons. Relationships between respondent age and IAT effects are presented as zero-order correlations (see Table 3). Older re-

Table 2

Implicit Association Test Effects Separated by Participant Gender or Ethnicity

\begin{tabular}{|c|c|c|c|c|c|c|}
\hline \multirow[b]{2}{*}{ Task } & \multicolumn{2}{|c|}{ Gender } & \multicolumn{4}{|c|}{ Ethnicity } \\
\hline & Women & Men & Asians & Blacks & Hispanics & Whites \\
\hline Race attitude (faces) & 0.65 & 0.76 & 0.78 & 0.16 & 0.66 & 0.83 \\
\hline Race attitude (names) & 0.81 & 0.99 & 0.91 & 0.28 & 0.88 & 1.04 \\
\hline Age attitude (faces) & 0.95 & 1.11 & 0.99 & 1.10 & 0.96 & 1.00 \\
\hline Age attitude (names) & 1.39 & 1.54 & 1.41 & 1.47 & 1.48 & 1.44 \\
\hline Gender-science & 0.73 & 0.72 & 0.64 & 0.66 & 0.67 & 0.74 \\
\hline Gender-career & 0.76 & 0.66 & 0.67 & 0.78 & 0.64 & 0.74 \\
\hline Self-esteem & 1.14 & 1.10 & 1.01 & 1.28 & 1.21 & 1.13 \\
\hline Math-arts attitude & 0.99 & 0.58 & 0.61 & 0.65 & 0.80 & 0.87 \\
\hline Election 2000 attitude & 0.23 & 0.02 & 0.29 & 0.73 & 0.26 & 0.08 \\
\hline
\end{tabular}

Note. All effects are presented in effect size form (Cohen's $d$ ). 
Table 3

Pearson's r Correlations Between IAT Effects and Methodological Variables, Participant Age, and Explicit Attitude or Stereotype

\begin{tabular}{lcc}
\hline \multicolumn{1}{c}{ Task } & $\begin{array}{c}\text { Respondent } \\
\text { age }\end{array}$ & $\begin{array}{c}\text { Explicit } \\
\text { preference }\end{array}$ \\
\hline Race attitude (faces) & -.01 & .17 \\
Race attitude (names) & .09 & .24 \\
Age attitude (faces) & .03 & .13 \\
Age attitude (names) & .11 & .08 \\
Gender-science & .09 & .21 \\
Gender-career & .09 & .17 \\
Self-esteem & .15 & .17 \\
Math-arts attitude & .11 & .47 \\
Election 2000 attitude & .06 & .52 \\
$\quad$ Average $r$ & .08 & .24 \\
\hline
\end{tabular}

Note. $\quad$ IAT $=$ implicit association test.

${ }^{\text {a }}$ Correlations for the Election 2000 task (Gore-Bush IATs only) were computed after partialing for pairing order. In that task, participants selected the presidential candidates to compare themselves. Comparisons with explicit preferences demonstrated that participants tended to select their preferred candidate first, confounding explicit preference with pairing order. Partialing for pairing order removed the effect of this tendency.

spondents tended to show slightly stronger effects than younger respondents across tasks (average $r=.08$; range $=-.01-.15$ ). Older respondents showed stronger links of male with science and female with liberal arts and stronger links of male with career and female with family than younger respondents. ${ }^{9}$ In addition, older participants tended to show stronger implicit bias against Black relative to White and old relative to young on the name versions of the task but not on the face versions.

\section{Overall Implicit-Explicit Effects and Their Correspondence}

Effect sizes were noticeably stronger on the implicit measure of attitude and belief than on the explicit measures on seven of the nine tasks (average $d$ for IAT $=0.84$, range $=0.14-1.42$; average $d$ for self-report $=0.39$, range $=0.17-$ 0.73; see Table 1). The two gender stereotype measures showed close correspondence between implicit and explicit measures.

Recent discussions of the relationship between implicit and explicit social cognition have emphasized dissociations between the two (Fazio, Sanbonmatsu, Powell, \& Kardes, 1986; Fazio et al., 1995; Greenwald \& Banaji, 1995).
At the demonstration Web site, across all tasks, implicit and explicit measures of attitude and stereotyping were, in general, positively correlated. This relationship ranged from small $(r=$ .08 for age names IAT) to moderate $(r=.24$ for race names IAT) to large ( $r=.52$ for Election 2000 [Bush-Gore] IAT; see Table 3). ${ }^{10}$ Implicit attitudes, as measured by the IAT, are reliably (albeit often only weakly) related to corresponding explicit attitudes. Yet these relationships are likely to be underestimated because of measurement error in the IAT (Cunningham, Preacher, \& Banaji, 2001). Though self-presentational biases (i.e., social desirability) are likely to play a partial role in predicting the strength of implicit-explicit relationships, many others (e.g., attitude elaboration) may also prove to be important predictors of implicit-explicit correspondence.

\section{General Discussion}

With data harvested from a demonstration Web site, we replicated laboratory studies showing that attitudes and stereotypes about groups exist in unconscious form, that is, relatively outside the ability to exert conscious control. In some cases, the magnitude of such effects mirrored those expressed consciously (e.g., gender-science stereotypes), but implicit biases were notably stronger than their explicit counterparts and were sometimes in contradiction to them (e.g., the dissociation between Black and White respondents on conscious versus unconscious attitudes). Findings like these reflect the propensity to consciously deny feelings and thoughts either because of social (external) pressures or personal (internal) standards. Differences between implicit and explicit attitudes do not suggest that one is accurate (or

\footnotetext{
${ }^{9}$ A possible alternative explanation holds that older respondents may show stronger IAT effects because they responded more slowly in the IAT overall. Although older respondents did perform the task more slowly than younger respondents, that slowness did not moderate the magnitude of their IAT effects.

${ }^{10}$ Implicit-explicit correlations from two tasks (both race tasks) were notably different between the full data set and the very conservative data set summarized in footnote 2 . The correlation between the IAT and the explicit measure on the race face task increased from .17 in the full data set to .29 in the conservative data set. Likewise, the correlation between the IAT and the explicit measure on the race name task increased from .24 to .30 .
} 
real) and the other is not. Rather, they suggest a form of mental (and often unrecognized) dissociation between implicit and explicit feelings and thoughts. Although recent research has shown that implicit and explicit attitudes can be in conflict, it is for future research to demonstrate the vicissitudes of each.

In one sense, the Web data showed a lack of moderating effects on the implicit attitude or belief that was measured. From young to old, male to female, Black to White, and conservative to liberal, implicit biases are not held by a select few but are readily observed among all social groups. Yet these data also show variation as a function of group membership and the constraints that culture imposes on individual attitudes. The implicit measures show such effects to a greater extent or in a manner that contradicts the findings from explicit measures. The standard in-group favorability typically captured on explicit measures alone, for example, is not visible on particular implicit measures. White Americans and young people show strong and robust liking for their own group. On the other hand, Black Americans show strong explicit liking for their group, and elderly people show more positive attitudes toward theirs (compared with younger individuals), but members of these groups do not show positive implicit in-group effects. Instead their own implicit attitudes reveal the influence of the negative attitudes held by the culture toward those groups. Although the exact origins of all forms of attitude and beliefs are not known, we regard implicit attitudes to reveal the deep influence of the immediate environment and the broader culture on internalized preferences and beliefs. Yet we regard the data from implicit measures to reflect an individual's own implicit attitudes and beliefs just as we do their more conscious ones. The learning context is culture, but the repository is the individual (Banaji, 2001).

Although implicit and explicit attitudes have been conceptualized as exclusive and unrelated measures of preference, these and other data demonstrate that implicit and explicit attitudes are more associated than previously assumed. The strength of this relationship varies by attitude object, and the moderators of this relationship are yet to be discovered. We know from our own recent research that not only are the reliability and convergent validity of implicit measures substantial (Cunningham et al., 2001), the relationship between explicit and implicit measures is also substantial, even though they constitute distinguishable factors (Cunningham et al., 2000).

With time, we hope that the present Web site, designed primarily for education and demonstration purposes, will contain full-scale experiments that will advance psychological science while educating public and, in the process, blur the distinction between research and teaching. We designed this site with the goal to disseminate and educate about our work on implicit social cognition. Our secondary goal was to gain experience with Internet-based research and to gather information about how best to address the multitude of conceptual and technological issues raised by this medium for scientific inquiry. We were unprepared for the overwhelming response the Web site received initially and continues to receive. We were gratified by the greater diversity of the sample that was obtained at the site (compared with our college samples), by the good fit of the Web data to those obtained in many laboratory experiments (see also Birnbaum, 1999), and by the fit of Web data to theory. Excitement about the enormous potential of the Internet should only facilitate the careful analysis of the costs and challenges as well as the benefits of the Internet as a tool for the behavioral sciences.

\section{References}

Banaji, M. R. (2001). Implicit attitudes can be measured. In H. L. Roediger, J. S. Nairne, I. Neath, \& A. Surprenant (Eds.), The nature of remembering: Essays in honor of Robert G. Crowder (pp. 117150). Washington DC: American Psychological Association.

Banaji, M. R., \& Greenwald, A. G. (1995). Implicit gender stereotyping in judgments of fame. Journal of Personality and Social Psychology, 68, 181198.

Banaji, M. R., \& Hardin, C. D. (1996). Automatic stereotyping. Psychological Science, 7, 136-141.

Banaji, M. R., Greenwald, A. G., \& Rosier, M. R. (1997). Implicit esteem: When collectives shape individuals. Paper presented at the Preconference on Self, Toronto, Canada.

Bargh, J. A., Chen, M., \& Burrows, L. (1996). Automaticity of social behavior: Direct effects of trait construct and stereotype activation on action. Journal of Personality and Social Psychology, 71, 230-244. 
Birnbaum, M. H. (1999). Testing critical properties of decision making on the Internet. Psychological Science, 10, 399-407.

Chartrand, T. L., \& Bargh, J. A. (1999). The chameleon effect: The perception-behavior link and social interaction. Journal of Personality and Social Psychology, 76, 893-910.

Cohen, J. (1988). Statistical power analysis for the behavioral sciences. Hillsdale, NJ: Erlbaum.

Cunningham, W. A., Nezlek, J. B., \& Banaji, M. R. (2000). Conscious and unconscious ethnocentrism: Revisiting the ideologies of prejudice. Unpublished manuscript.

Cunningham, W. A., Preacher, K. J., \& Banaji, M. R. (2001). Implicit attitude measures: Consistency, stability, and convergent validity. Psychological Science, 12, 163-170.

Devine, P. G. (1989). Stereotypes and prejudice: Their automatic and controlled components. Journal of Personality and Social Psychology, 56, 5-18.

Dovidio, J. F., Kawakami, K., Johnson, C., Johnson, B., \& Howard, A. (1997). On the nature of prejudice: Automatic and controlled processes. Journal of Experimental Social Psychology, 33, 510-540.

Fazio, R. H., Jackson, J. R., Dunton, B. C., \& Williams, C. J. (1995). Variability in automatic activation as an unobstrusive measure of racial attitudes: A bona fide pipeline? Journal of Personality and Social Psychology, 69, 1013-1027.

Fazio, R. H., Sanbonmatsu, D. M., Powell, M. C., \& Kardes, F. R. (1986). On the automatic activation of attitudes. Journal of Personality and Social Psychology, 50, 229-238.

Gilbert, D. T., \& Hixon, J. G. (1991). The trouble of thinking: Activation and application of stereotypic beliefs. Journal of Personality and Social Psychology, 60, 509-517.

Greenwald, A. G., \& Banaji, M. R. (1995). Implicit social cognition: Attitudes, self-esteem, and stereotypes. Psychological Review, 102, 4-27.

Greenwald, A. G., McGhee, D. E., \& Schwartz, J. L. K. (1998). Measuring individual differences in implicit cognition: The Implicit Association Test. Journal of Personality and Social Psychology, 74, 1464-1480.
Greenwald, A. G., Nosek, B. A., \& Banaji, M. R. (2002). Scoring procedures to improve the usefulness of Implicit Association Test measures. Unpublished manuscript.

Jost, J. T., \& Banaji, M. R. (1994). The role of stereotyping in system-justification and the production of false consciousness. British Journal of Social Psychology, 33, 1-27.

Katz, I., \& Hass, R. G. (1988). Racial ambivalence and American value conflict: Correlational and priming studies of dual cognitive structures. Journal of Personality and Social Psychology, 55, 893905.

Katz, I., Wackenhut, J., \& Hass, R. G. (1986). Racial ambivalence, value duality, and behavior. In J. F. Dovidio \& S. L. Gaertner (Eds.), Prejudice, discrimination, and racism (pp. 35-59). Orlando, FL: Academic Press.

Mitchell, J. A., Nosek, B. A., \& Banaji, M. R. (2001). Contextual variation in implicit evaluation. Manuscript submitted for publication.

Moskowitz, G. B., Gollwitzer, P. M., Wasel, W., \& Schaal, B. (1999). Preconscious control of stereotype activation through chronic egalitarian goals. Journal of Personality and Social Psychology, 77, 167-184.

National Science Foundation. (1996). Women, minorities, and persons with disabilities in science and engineering: 1996 (NSF Publication No. 96311). Arlington, VA: Author.

Nosek, B. A., Banaji, M. R., \& Greenwald, A. G. (in press). Math $=$ male, me $=$ female, therefore math $\neq$ me. Journal of Personality and Social Psychology.

Perdue, C. W., \& Gurtman, M. B. (1990). Evidence for the automaticity of ageism. Journal of Experimental Social Psychology, 26, 199-216.

Phelps, E. A., O’Connor, K. J., Cunningham, W. C., Funayama, E. S., Gatenby, J. C., Gore, J. C., \& Banaji, M. R. (2001). Performance on indirect measures of race evaluation predicts amygdala activation. Journal of Cognitive Neuroscience, 12, 729-738.

Wang, C. S., \& Banaji, M. R. (1999). [Implicit gender stereotypes about career and family.] Unpublished data. 


\section{Appendix A}

Labels and Stimuli for Implicit Measures

\begin{tabular}{|c|c|}
\hline Label & Stimuli \\
\hline \multicolumn{2}{|l|}{ Race attitude (name) } \\
\hline Black & Tyrone, Malik, Jamal, Leroy, Temeka, Latisha, Tawanda, Shaniqua \\
\hline White & Chip, Brad, Walter, Ralph, Heather, Betsy, Peggy, Colleen \\
\hline \multicolumn{2}{|l|}{ Age attitude (name) } \\
\hline Old & Ethel, Bernice, Gertrude, Agnes, Cecil, Wilbert, Mortimer, Edgar \\
\hline Young & Tiffany, Michelle, Cindy, Kristy, Brad, Eric, Joey, Billy \\
\hline \multicolumn{2}{|r|}{ 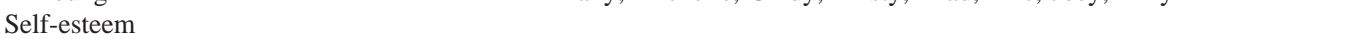 } \\
\hline Self & I, Me, Mine, Myself \\
\hline Other & They, Them, Their, Theirs \\
\hline \multicolumn{2}{|l|}{ Gender-career stereotype } \\
\hline Male & John, Paul, Mike, Kevin, Steve, Greg, Jeff, Bill \\
\hline Female & Amy, Joan, Lisa, Sarah, Diana, Kate, Ann, Donna \\
\hline Career & $\begin{array}{l}\text { Executive, Management, Professional, Corporation, Salary, Office, } \\
\text { Business, Career }\end{array}$ \\
\hline Family & Home, Parents, Children, Family, Cousins, Marriage, Wedding, Relatives \\
\hline \multicolumn{2}{|l|}{ Gender-science stereotype } \\
\hline Male & Male, Man, Boy, Brother, He, Him, His, Son \\
\hline Female & Female, Woman, Girl, Sister, She, Her, Hers, Daughter \\
\hline Science & $\begin{array}{l}\text { Chemistry, Physics, Biology, Biophysics, Engineering, Astronomy, } \\
\text { Biochemistry, Neuroscience }\end{array}$ \\
\hline Liberal arts & Philosophy, Arts, Humanities, History, Spanish, English, Latin, Music \\
\hline \multicolumn{2}{|l|}{ Math attitude } \\
\hline Math & $\begin{array}{l}\text { Math, Algebra, Geometry, Calculus, Equations, Computation, Numbers, } \\
\text { Addition }\end{array}$ \\
\hline Arts & Poetry, Art, Dance, Literature, Novel, Symphony, Drama, Sculpture \\
\hline \multicolumn{2}{|l|}{ Race attitude (face) } \\
\hline African American & 6 morphed faces ( 3 male, 3 female) \\
\hline Euro-American & 6 morphed faces ( 3 male, 3 female) \\
\hline \multicolumn{2}{|l|}{ Age attitude (face) } \\
\hline Old & 6 morphed faces ( 3 male, 3 female) \\
\hline Young & 6 morphed faces ( 3 male, 3 female) \\
\hline \multicolumn{2}{|l|}{ Election 2000 preferences } \\
\hline (Candidate A) & Label $=$ last name of the selected candidate \\
\hline (Candidate B) & 4 stimuli $=2$ faces, first and last name, last name \\
\hline \multicolumn{2}{|c|}{ Evaluative items for all attitude measures } \\
\hline Good & Joy, Love, Peace, Wonderful, Pleasure, Friend, Laughter, Happy \\
\hline $\mathrm{Bad}$ & Agony, Terrible, Horrible, ${ }^{\mathrm{a}}$ Nasty, Evil, War, Awful, Failure, Death ${ }^{\mathrm{a}}$ \\
\hline
\end{tabular}

${ }^{\text {a }}$ In November 1999, the stimulus item Death was removed and replaced with Horrible. 


\section{Appendix B}

Questionnaire Items for Each Task

All tasks

- Explicit preference $(5=$ point Likert rating)

- Sex

- Age

- Ethnicity

- State/country of residence

- How many IATs have you previously performed?

Race (Name), Age (Name), Self-Esteem, Math Attitude, Gender-Career Stereotype

- How many times have you previously performed this IAT?

Race (Face), Age (Face), Gender-Science Stereotype, Election 2000

- Education

- Population of the city where you have spent most of your life

\section{Race (Face)}

- Which is your dominant hand? (right, left, ambidextrous)
- What percentage of the people you interact with (at work, at school, socially) are Black?

- What percentage of the people you interact with (at work, at school, socially) are White?

- Politically speaking, do you consider yourself to be ... (strongly liberal, liberal, moderate, conservative, strongly conservative)

\section{Age (Face)}

- I think of myself as ... (very young, young, middle aged, old, very old)

\section{Gender-Science Stereotype}

- Please rate your attitude toward science. (strongly like, like, neither like nor dislike, dislike, strongly dislike)

- Please rate your attitude toward liberal arts. (strongly like, like, neither like nor dislike, dislike, strongly dislike)

\section{Election 2000}

- I think of myself as a... (Democrat, Republican, Reform, other)

- Are you presently registered to vote? 